\title{
Characteristics and Outcomes of COVID-19 Patients Admitted to Intensive Care Units in a Large Health System in Western Pennsylvania
}

Adeel Nasrullah ${ }^{1}$, Thejus Jayakrishnan ${ }^{2}$, Patrick Wedgeworth ${ }^{2}$, Melissa Mosley ${ }^{2}$, Kirtivardhan Vashistha 2 , Aaron Haag ${ }^{2}$, Veli Bakalov ${ }^{2}$, Abhishek Chaturvedi ${ }^{2}$, Susan Manzi ${ }^{3}$, Anastasios Kapetanos ${ }^{2}$

1. Pulmonary and Critical Care Medicine, Allegheny Health Network, Pittsburgh, USA 2. Internal Medicine, Allegheny Health Network, Pittsburgh, USA 3. Rheumatology, Allegheny Health Network, Pittsburgh, USA

Corresponding author: Adeel Nasrullah, adeel.shifa@gmail.com

\section{Abstract \\ Importance}

Despite growing literature, there is still limited understanding of factors that can predict outcomes in coronavirus disease 2019 (COVID-19) patients who require intensive care.

\section{Objective}

To evaluate the characteristics of COVID-19 patients admitted to the intensive care unit (ICU) and identify their associations with outcomes.

\section{Background}

There are limited data on the outcomes in COVID-19 patients in Pennsylvania.

\section{Design}

Retrospective study

\section{Setting}

Intensive care units in an academic health system in Western Pennsylvania.

\section{Participants}

Patients with reverse transcriptase-polymerase chain reaction (RT-PCR)-confirmed COVID-19 admitted to ICUs as direct admission or transfers from regular floors between March 1, 2020, and April 30, 2020.

Review began 06/29/2021 Review ended 07/13/2021 Published 07/22/2021

๑) Copyright 2021 Nasrullah et al. This is an open access article distributed under the terms of the Creative Commons Attribution License CC-BY 4.0., which permits unrestricted use, distribution, and reproduction in any medium, provided the original author and source are credited.

\section{Main outcome(s) and measure(s)}

The primary outcome was inpatient mortality. Secondary outcomes included complications during ICU stay, hospital length of stay, discharge disposition, and the need for oxygen at discharge. Categorical variables are described as frequencies and continuous variables as median with interquartile range (IQR). Regression modeling was used to identify the predictors of inpatient mortality in these patients. P-value $<0.05$ was considered statistically significant. Analysis was performed using Stata version 15.1 (StataCorp, College Station, Texas).

\section{Results}

The cohort included 58 consecutive patients, with a median age of 62 years (IQR 54-73), 63.8\% of which were male. On presentation, constitutional symptoms were the most common (91.4\%), followed by lower respiratory tract symptoms (87.9\%). Tachypnea (65.5\%) and hypoxia (67.2\%) were the most common abnormal vital signs at presentation. Common comorbidities were cardiovascular disease (74.1\%), obesity (53.5\%), and diabetes (39.7\%). The median Acute Physiology and Chronic Health Evaluation (APACHE) score on admission to ICU was 11 (IQR 8.5-17.5). The major complications included acute respiratory distress syndrome (ARDS) $50.0 \%$, shock $41.4 \%$, and acute kidney injury $41.4 \%$. The proportion of patients who underwent mechanical ventilation, required vasopressors, or were on renal replacement therapy were $58.6 \%$, $41.4 \%$, and $10.3 \%$, respectively. Overall mortality was 32.8\%. Age, Charlson-comorbidity index, tachypnea, lymphopenia at presentation, high APACHE score, shock, ARDS, mechanical ventilation, and steroid use were significantly associated with mortality. Of the patients who survived their ICU stay, $63.2 \%$ were discharged home and $44.7 \%$ had a new oxygen requirement at discharge.

\section{Conclusion and relevance}


Our study reports high mortality in COVID-19 patients requiring ICU care in Western Pennsylvania. Identifying factors associated with poor prognosis could help risk-stratify these patients. Prospective studies are needed to assess whether early risk stratification and triaging result in improved outcomes.

Categories: Internal Medicine, Infectious Disease

Keywords: covid-19, sars-cov-2, viral pneumonia, pandemic, icu, mortality

\section{Introduction}

Since its emergence, coronavirus disease 2019 (COVID-19) has remained a global health crisis and continues to impart significant social, psychological, and logistical burdens on individuals and health care systems [12]. The United States has reported the largest number of cases, and as of July 13, 2021, there were 33,726,363 COVID-19 cases with 605,140 deaths [3]. Robust research has resulted in a better understanding of the natural history of the disease, characteristics of patients, and predictors of outcomes. Despite this, there is still a need for more evidence data regarding characteristics and outcomes of those admitted to intensive care units (ICUs), particularly in the United States. Preliminary studies suggest that up to $6 \%$ of COVID-9 patients in the US required ICU admission, conferring the highest mortality to this group [4].

Allegheny Health Network (AHN) is one of the largest consortiums of academic urban and non-academic rural hospitals in Western Pennsylvania and has been at the forefront of COVID-19 in Pennsylvania [5-7]. Up to July 13, 2021, the state of Pennsylvania reported 1,214,654 COVD-19 cases with 27,769 deaths, however, characteristics specific to severely ill patients have not been published [8]. In this context; the present study was conducted to describe the initial experience with regards to the clinical characteristics and outcomes for patients with COVID-19 admitted to ICUs across a large health system. Factors associated with mortality in this population were also identified.

\section{Materials And Methods}

A retrospective study was conducted of consecutive patients diagnosed with a COVID-19 by real-time polymerase chain reaction (RT-PCR) test via nasal swab admitted to intensive care units (ICUs) between March 1, 2020, and April 30, 2020. The cohort included patients transferred to ICU for escalation of care and direct admissions from the emergency department. For patients with readmissions, only index hospitalization due to COVID-19 is included in this study. Patient disposition to the ICU was determined by the need for mechanical ventilation, vasopressors, or the clinical judgment of providers. The severity of illness was determined by sequential organ assessment failure (SOFA) and the acute physiology and chronic health evaluation 2 (APACHE 2) scoring system.

The AHN Institutional Review Board approved the study and waived the need for informed consent. Deidentified data collection was performed from the electronic health record system (Epic) and included patient demographics, medical history, home medications, clinical presentation characteristics, and treatment strategies. The symptoms were grouped into the following categories: constitutional (anorexia, fever, chills, myalgia or arthralgia, and fatigue), upper respiratory tract (sore throat, nasal congestion, anosmia, and dysgeusia), lower respiratory tract (cough, dyspnea, sputum, hemoptysis), and others (chest pain, palpitation, lightheadedness, syncope, abdominal pain, diarrhea, nausea or vomiting, conjunctival injection, altered mental status, headache, and muscle weakness). Headache was included in the "others" category, as it could be both a constitutional and a neurological symptom. Comorbidities were classified as follows: cardiovascular (congestive heart failure, coronary artery disease, arrhythmias, valvular disease, hypertension, pulmonary hypertension, peripheral vascular disease, other cardiac diseases), pulmonary (chronic obstructive pulmonary disease, asthma, interstitial lung disease, pulmonary hypertension, other lung diseases), renal disease (chronic kidney disease, including end-stage renal disease requiring dialysis). Charlson-comorbidity index (CCI) was also calculated for analysis.

The primary outcome was mortality during the hospital stay. Secondary outcomes included complications during the ICU stay (acute respiratory distress syndrome (ARDS), shock, acute kidney injury, deep venous thrombosis (DVT) or pulmonary embolism (PE), bleeding, and cardiac arrest), hospital length of stay, discharge disposition (home versus assisted care facility), and oxygen need at discharge. ARDS was defined using the Berlin Criteria (presence of acute respiratory failure with bilateral pulmonary infiltrates, a ratio of arterial oxygen tension to a fraction of inspired oxygen $<300$ with $>5 \mathrm{~cm}$ water of positive-end expiratory pressure, and absence of cardiogenic pulmonary edema) [9]. Acute kidney injury was defined as an absolute increase in serum creatinine of more than or equal to $0.3 \mathrm{mg} / \mathrm{dl}$, a percentage increase in serum creatinine of more than or equal to $50 \%$ (1.5-fold from baseline), or a reduction in urine output (documented oliguria of less than $0.5 \mathrm{ml} / \mathrm{kg}$ per hour for more than six hours) [10].

Categorical outcomes are described in percentages and continuous variables with median and interquartile range (IQR). Univariate logistic regression analysis for mortality was performed for variables with at least 20 observations. Multivariate regression was not performed due to the small sample size and established interactions among different variables resulting in a model that may not add meaningful additional information to the existing results. Results are represented as odds ratio (OR) with a $95 \%$ confidence interval 


\section{Cureus}

(95\% CI) for applicable variables. Statistical tests were two-tailed, and statistical significance was defined as p-value $<.05$. Analyses were performed using Stata version 15.1 (StataCorp, College Station, Texas). Since the analyses have not been adjusted for multiple comparisons and is prone to type-I error, the findings should be interpreted as exploratory.

\section{Results}

\section{Characteristics of patients admitted to ICU}

Patient characteristics are outlined in Table 1. The cohort included 58 patients, with a median age of 62 years (IQR 54-73), and included 37 (63.8\%) males. The majority of patients were non-Hispanic White (60.3\%) followed by non-Hispanic Black (29.3\%). Constitutional symptoms were reported by $91.4 \%$ while $87.9 \%$ reported lower respiratory tract symptoms, and $20.7 \%$ reported upper respiratory symptoms. Common comorbidities included cardiovascular disease (74.1\%), obesity (53.5\%), and diabetes (39.7\%). The median CCI was 1 (IQR 0-2).

\begin{tabular}{|c|c|}
\hline Characteristics & Value $^{a}$ \\
\hline Age (in years) & $62(54-73)$ ye \\
\hline Male & $37(63.8 \%)$ \\
\hline \multicolumn{2}{|l|}{ Race } \\
\hline Non-Hispanic White & $35(60.3 \%)$ \\
\hline Non-Hispanic Black & $17(29.3 \%)$ \\
\hline Hispanic & $2(3.5 \%)$ \\
\hline Other/Unknown & $4(6.9 \%)$ \\
\hline \multicolumn{2}{|l|}{ Symptoms } \\
\hline Constitutional & $53(91.4 \%)$ \\
\hline Lower respiratory symptoms & $51(87.9 \%)$ \\
\hline Upper respiratory symptoms & $12(20.7 \%)$ \\
\hline Other systems/nonspecific symptoms & $38(65.5 \%)$ \\
\hline \multicolumn{2}{|l|}{ Comorbidities } \\
\hline Cardiovascular & $43(74.1 \%)$ \\
\hline Obesity $\left(\mathrm{BMl}>30 \mathrm{~kg} / \mathrm{m}^{2}\right)$ & $31(53.5 \%)$ \\
\hline Diabetes & $23(39.7 \%)$ \\
\hline Pulmonary & $9(15.5 \%)$ \\
\hline Renal & $8(13.8 \%)$ \\
\hline Other & $23(39.7 \%)$ \\
\hline ACE/ARB & $22(37.9 \%)$ \\
\hline \multicolumn{2}{|l|}{ Smoking } \\
\hline Active smoker & $23(39.7 \%)$ \\
\hline \multicolumn{2}{|l|}{ Admission Vitals } \\
\hline Temperature $>38$ degree-Celsius & $17(29.3 \%)$ \\
\hline Heart rate $>100$ beats per minute & $27(46.6 \%)$ \\
\hline Respiratory rate $>20$ breaths per minute & $38(65.5 \%)$ \\
\hline Systolic blood pressure $(\mathrm{mm} \mathrm{Hg})<90$ & $1(1.7 \%)$ \\
\hline Hypoxia at presentation & $39(67.2 \%)$ \\
\hline Abnormal CXR $(n=52)$ & $44(84.6 \%)$ \\
\hline
\end{tabular}




\section{Cureus}

Hospital admission Abnormal Lab Findings

AST/ALT $>40 \mathrm{U} / \mathrm{L}$

$36(62.1 \%)$

Absolute lymphocyte count $<0.60 \mathrm{k} / \mathrm{mcl}$

$17(29.3 \%)$

Hospital admission labs

White blood cell count $\mathrm{k} / \mathrm{mcl}$

$6.8(5.8-10.5)$

Creatinine $\mathrm{mg} / \mathrm{dl}$

$1.1(0.8-1.5)$

Procalcitonin $\mathrm{ng} / \mathrm{ml}(\mathrm{n}=44)$

$0.2(0.1-0.4)$

Lactate dehydrogenase $\mathrm{U} / \mathrm{I}(\mathrm{n}=34)$

503 (377-778)

D-dimer $\mathrm{mg} / \mathrm{ml}(\mathrm{n}=31)$

$1.5(1.1-3.8)$

Pro-brain natriuretic peptide $\mathrm{pg} / \mathrm{ml}(\mathrm{n}=29)$

$291(131-2557)$

C-reactive protein $\mathrm{mg} / \mathrm{dl}(\mathrm{n}=28)$

$11.9(6.4-17.4)$

Erythrocyte sedimentation rate $\mathrm{mm} /$ hour $(\mathrm{n}=6)$

$51(29-84)$

ICU Admission severity of illness

APACHE 2 (40)

$11(8.5-17.5)$

SOFA (44)

Treatment Strategies received

Hydroxychloroquine

$45(77.6 \%)$

Empiric antibiotics

$44(75.9 \%)$

Mechanical ventilation

$34(58.6 \%)$

Vasopressor use

$24(41.4 \%)$

Steroids

$20(34.5 \%)$

Neuromuscular blockade use

$11(19.0 \%)$

Proning

$10(37.9 \%)$

Blood transfusion

9 (15.5\%)

Renal replacement therapy

$5(8.6 \%)$

Plasmapheresis

$4(6.9 \%)$

Intravenous immunoglobulin

Mechanical circulatory support

$1(1.7 \%)$

\section{TABLE 1: Characteristics for patients admitted with COVID-19 to the ICU}

a Continuous variables are reported as median (IQR) and categorical variables as frequency (percentage).

IQR=Interquartile Range; HCP=Healthcare Provider; $\mathrm{BMI}=$ Body Mass Index; $\mathrm{ACE}=$ Angiotensin-Converting Enzyme; $\mathrm{ARB}=\mathrm{Angiotensin}$ Receptor Blocker; CXR=Chest X-Ray; AST=Aspartate Aminotransferase; ALT=Alanine Transaminase; APACHE 2=Acute Physiology and Chronic Health Evaluation 2; SOFA=Sequential Organ Assessment Failure

Upon presentation, $65.5 \%$ patients were tachypneic, $67.2 \%$ were hypoxic, and $46.6 \%$ were tachycardic. Important admission laboratory abnormalities included transaminitis in $62.1 \%$ of patients, lymphopenia in $29.3 \%$, and abnormal chest X-ray in $84.6 \%$ of patients among those who had the test performed.

The most common abnormal chest X-ray finding was interstitial opacities in $86.3 \%$ (38 of 44 patients). Fourteen patients had admission chest computed tomography (CT) and were $100 \%$ abnormal. Inflammatory markers (C-reactive protein (CRP), erythrocyte sedimentation rate (ESR), D-dimer) were not obtained in all patients but were elevated in those measured. The median APACHE 2 score at admission to ICU was 11 (IQR 8.5-17.5), and the sequential assessment of organ failure score was 3 (IQR 2-5). Table 1 summarizes the 


\section{Cureus}

characteristics of patients admitted to ICU.

\section{ICU treatments}

Following admission to the ICU, 58.6\% required mechanical ventilation, 41.4\% required vasopressor support, 8.6\% needed renal replacement therapy, and one patient needed mechanical circulatory support (Table 1). Pharmacological treatment strategies included hydroxychloroquine in $77.6 \%$, empiric antibiotics in $75.9 \%$, steroids in $34.5 \%$, plasmapheresis in $6.9 \%$, and intravenous immunoglobulin in $5.2 \%$ of patients.

\section{ICU outcomes and predictors of inpatient mortality}

Overall, inpatient mortality was $32.8 \%$ in our cohort. The major complications included: ARDS in $50.0 \%$, shock in $41.4 \%$, AKI in $41.4 \%$, venous thromboembolism (DVT/PE) in $7.0 \%$, and bleeding in $6.9 \%$ patients. The median total length of stay for this cohort was 11.5 days (IQR 5-20). Median length of stay in the ICU was six days (IQR 3-16) to 13 days (4-21) for survivors versus five days (IQR 2-11) for non-survivors. Among survivors, $63.2 \%$ were discharged home and $34.2 \%$ to assisted care facility. Additionally, $44.7 \%$ patients had a new oxygen requirement upon discharge. On univariate regression modeling, age [OR 1.05(1.01-1.11) pvalue $=0.015$ ], CCI [OR 1.37(1.02-1.82) $\mathrm{p}$-value $=0.032$ ], tachypnea [OR 17.1(2.1-141.0 $\mathrm{p}$-value $=0.008]$ and lymphopenia [OR 3.9(1.2-13.0) $\mathrm{p}$-value=0.028] on admission, APACHE-2 score [OR 1.13(1.03-1.24) $\mathrm{p}$ value $=0.002$ ], steroid use [OR 4.6(1.4-14.9) $\mathrm{p}$-value $=0.010$ ], development of ARDS [OR 4.5(1.3-15.0) $\mathrm{p}$ value $=0.015]$, and shock [OR $8.1(2.3-28.3) \mathrm{p}=0.004]$ were found to be associated with inpatient mortality. ICU outcomes and predictors of inpatient mortality are summarized in Tables 2-3.

\begin{tabular}{|l|l|}
\hline Outcomes & Value a \\
\hline Mortality & $19(32.8 \%)$ \\
\hline Complications & \\
ARDS & $29(50.0 \%)$ \\
Shock & $24(41.4 \%)$ \\
\hline Acute kidney injury & $24(41.4 \%)$ \\
\hline DVT/PE & $4(6.9 \%)$ \\
\hline Bleeding & $4(6.9 \%)$ \\
\hline Cardiac arrest & $3(5.2 \%)$ \\
\hline Hospital length of stay, days & $11.5(5-20)$ \\
\hline Discharge disposition $(n=38)^{\mathrm{b}}$ & \\
\hline Home & $24(63.2 \%)$ \\
\hline Assisted care facility & $13(34.2 \%)$ \\
\hline Outside hospital & $1(2.6 \%)$ \\
\hline New oxygen at discharge & $17(44.7 \%)$ \\
\hline
\end{tabular}

\section{TABLE 2: Outcomes for patients admitted to ICU}

a Continuous variables are reported as median (IQR) and categorical variables as frequency (percentage).

IQR=Interquartile Range; HCP=Healthcare Provider; ARDS=Acute Respiratory Distress Syndrome; DVT=Deep Venous Thrombosis; PE=Pulmonary Embolism

b Alive at discharge

\begin{tabular}{|c|c|c|c|c|}
\hline Characteristics & Survivors $(n=39)$ Value ${ }^{a}$ & Non-Survivors $(n=19)$ Value $^{a}$ & Odds ratio $(95 \% \mathrm{Cl})$ & P-value \\
\hline Age (in years) & $61((54-67)$ & $65(60-79)$ & $1.05(1.01-1.11)$ & 0.015 \\
\hline Male & 23 (59.0\%) & 14 (73.7\%) & - & 0.26 \\
\hline
\end{tabular}




\section{Cureus}

\begin{tabular}{|c|c|c|c|c|}
\hline \multicolumn{5}{|l|}{ Race } \\
\hline Non-Hispanic White & $23(59.0 \%)$ & $12(63.2 \%)$ & Reference & \\
\hline Non-Hispanic Black & $11(28.2 \%)$ & $6(31.6 \%)$ & - & 0.94 \\
\hline Hispanic & $2(5.1 \%)$ & 0 & Not performed & \\
\hline Other/Unknown & $3(7.7 \%)$ & $1(5.3 \%)$ & - & 0.71 \\
\hline \multicolumn{5}{|l|}{ Symptoms } \\
\hline Constitutional & $38(97.4 \%)$ & $15(79.0 \%)$ & $0.10(0.01-0.95))$ & 0.02 \\
\hline Lower respiratory symptoms & $12(30.8 \%)$ & 17 (89.5\%) & & 0.80 \\
\hline Upper respiratory symptoms & $34(87.2 \%)$ & 0 & Not performed & \\
\hline Other systems/nonspecific symptoms & $26(66.7 \%)$ & $12(63.2 \%)$ & & \\
\hline \multicolumn{5}{|l|}{ Comorbidities } \\
\hline Cardiovascular & $27(69.2 \%)$ & $16(84.2 \%)$ & - & 0.21 \\
\hline Obesity $\left(\mathrm{BMl}>30 \mathrm{~kg} / \mathrm{m}^{2}\right)$ & $22(56.4 \%)$ & $9(47.4 \%)$ & - & 0.52 \\
\hline Diabetes & $15(38.5 \%)$ & $8(42.1 \%)$ & - & 0.79 \\
\hline Pulmonary & $6(15.4 \%)$ & $3(15.8 \%)$ & - & 0.96 \\
\hline Renal & $4(10.3 \%)$ & $4(21.1 \%)$ & - & 0.28 \\
\hline Other & 13 (33.3\%) & $10(52.6 \%)$ & - & 0.16 \\
\hline $\mathrm{CCl}$ & $1(0-1)$ & $1(0-4)$ & $1.37(1.02-1.82)$ & 0.032 \\
\hline ACE/ARB & $24(61.5 \%)$ & $6(31.6 \%)$ & - & 0.48 \\
\hline \multicolumn{5}{|l|}{ Smoking } \\
\hline Active smoker & $16(41.0 \%)$ & $7(36.8 \%)$ & - & 0.80 \\
\hline \multicolumn{5}{|l|}{ Admission Vitals } \\
\hline Temperature $>38$ degree-Celsius & $11(28.2 \%)$ & $6(31.6 \%)$ & - & 0.79 \\
\hline Heart rate $>100$ beats per minute & $16(41.0 \%)$ & $11(57.9 \%)$ & - & 0.22 \\
\hline Respiratory rate $>20$ breaths per minute & $20(51.3 \%)$ & $18(94.7 \%)$ & $17.1(2.1-141.0)$ & 0.008 \\
\hline Systolic blood pressure $(\mathrm{mm} \mathrm{Hg})<90$ & 0 & $1(5.3 \%)$ & Not performed & \\
\hline Hypoxia at presentation & $24(61.5 \%)$ & $15(79.0 \%)$ & - & 0.17 \\
\hline Abnormal CXR & $30(83.3 \%)$ & $14(87.5 \%)$ & & 0.70 \\
\hline \multicolumn{5}{|l|}{ Hospital admission Abnormal Lab Findings } \\
\hline AST/ALT>40 U/L & $22(56.4 \%)$ & $14(73.7 \%)$ & $2.2(0.7-7.2)$ & 0.20 \\
\hline Absolute lymphocyte count $<0.60 \mathrm{k} / \mathrm{mcl}(\mathrm{n}=57)$ & $8(20.5 \%)$ & $9(50 \%)$ & $3.9(1.2-13.0)$ & 0.028 \\
\hline \multicolumn{2}{|l|}{ Hospital admission labs } & . & & \\
\hline White blood cell count $\mathrm{k} / \mathrm{mcl}$ & $7.0(5.8-10.0)$ & $6.7(5.0-12.5)$ & - & 0.50 \\
\hline Creatinine $\mathrm{mg} / \mathrm{dl}$ & $1.0(0.8-1.3)$ & $1.5(0.9-2.0)$ & - & 0.07 \\
\hline Lactate dehydrogenase U/I $(n=34)$ & $600(353-845)$ & $488(395-687)$ & - & 0.45 \\
\hline D-dimer mg/ml (n=31) & $1.3(1.0-2.1)$ & $2.4(1.5-5.2)$ & - & 0.13 \\
\hline Pro-brain natriuretic peptide pg/ml $(\mathrm{n}=29)$ & $219(67-408)$ & $2152(297.5-6202.5)$ & - & 0.08 \\
\hline C-reactive protein mg/dl $(\mathrm{n}=28)$ & $10.4(6.2-13.6)$ & $16.7(10.5-26.7)$ & - & 0.13 \\
\hline Erythrocyte Sedimentation Rate mm/hr (n=6) & $29.0(9-42)$ & $84(60-123)$ & Not performed & \\
\hline
\end{tabular}




\section{Cureus}

\begin{tabular}{|c|c|c|c|c|}
\hline APACHE 2 (40) & $10(7-11)$ & $16(11-25)$ & $1.13(1.03-1.24)$ & 0.002 \\
\hline \multicolumn{5}{|l|}{ Treatment strategies received } \\
\hline Mechanical ventilation & $16(41.0 \%)$ & $18(94.7 \%)$ & $25.9(3.1-213.9)$ & $<0.0005$ \\
\hline $\mathrm{HCQ}$ & $30(76.9 \%)$ & $15(79.0 \%)$ & - & 0.86 \\
\hline Empiric antibiotics & $28(71.8 \%)$ & $16(84.2 \%)$ & - & 0.29 \\
\hline Pressors & $10(25.6 \%)$ & $14(73.7 \%)$ & $8.1(2.3-28.3)$ & 0.004 \\
\hline Steroids & $9(23.1 \%)$ & 11 (57.9\%) & $4.6(1.4-14.9)$ & 0.010 \\
\hline Neuromuscular blockade use & $7(18.4 \%)$ & $4(22.2 \%)$ & Not performed & \\
\hline Proning & $11(28.2 \%)$ & $11(67.9 \%)$ & $1.0(0.9-1.2)$ & 0.41 \\
\hline Blood transfusion & $3(7.7 \%)$ & 6 (31.6\%) & Not performed & \\
\hline Renal replacement therapy & $2(5.3 \%)$ & $3(15.8 \%)$ & Not performed & \\
\hline Plasmapheresis & $1(2.6 \%)$ & $3(15.8 \%)$ & Not performed & \\
\hline Intravenous immunoglobulin & $2(5.1 \%)$ & $1(5.3 \%)$ & Not performed & \\
\hline Mechanical circulatory support & $1(2.6 \%)$ & $18(94.7 \%)$ & Not performed & \\
\hline \multicolumn{5}{|l|}{ Complications } \\
\hline ARDS & $15(38.5 \%)$ & $14(73.7 \%)$ & $4.5(1.3-15.0)$ & 0.015 \\
\hline Shock & $10(25.6 \%)$ & $14(73.7 \%)$ & $8.1(2.3-28.3)$ & 0.0004 \\
\hline Acute kidney injury & $13(33.3 \%)$ & $11(57.9 \%)$ & $2.8(0.9-8.5)$ & 0.08 \\
\hline DVT/PE & $2(5.1 \%)$ & 0 & Not performed & \\
\hline Bleeding & 0 & $4(21.1 \%)$ & Not performed & \\
\hline Cardiac arrest & 0 & $3(15.8 \%)$ & Not performed & \\
\hline
\end{tabular}

\section{TABLE 3: Significant factors associated with mortality of patients admitted to ICU}

a Continuous variables are reported as median (IQR) and categorical variables as frequency (percentage).

IQR=Interquartile Range; $\mathrm{HCP}=$ Healthcare Provider; $\mathrm{CCl}=$ Charlson-Comorbidity Index; $\mathrm{BMI}=$ Body Mass Index; $\mathrm{ACE}=\mathrm{Angiotensin}-\mathrm{Converting}$ Enzyme; ARB=Angiotensin Receptor Blocker; CXR=Chest X-Ray; AST=Aspartate Aminotransferase; ALT=Alanine Transaminase; APACHE 2=Acute Physiology and Chronic Health Evaluation 2; $\mathrm{HCQ}=\mathrm{Hydroxychloroquine}$

\section{Discussion}

In this retrospective study of 58 COVID-19 patients admitted to the ICU, our findings are consistent with prior evidence from institutions within and outside of the United States as described below.

The cohort was predominantly male and older, similar to other studies [11-15]. The majority of patients had constitutional and lower respiratory tract symptoms at presentation, and more than $60 \%$ had respiratory distress at presentation [11-12,14,16-17]. Similarly, the cohort had abnormally elevated levels of inflammatory markers (D-dimer, CRP, ESR) at presentation. A definitive comparison of biomarkers in terms of risks for ICU admission or mortality cannot be made, as it was recognized late in the study period that admission and daily levels of these biomarkers were warranted in all patients [13]. Future studies have reported that older patients, the presence of type-2 diabetes, cancer, in-hospital complications (such as acute kidney injury, sepsis/septic shock, multiorgan dysfunction), and higher D-dimer > 5,000 ng/mL are associated with higher mortality. Although the prevalence of lymphocytopenia is lower than previously reported (63\%-70\%), it is associated with increased mortality [11,13,16-19]. Our study also found a statistically significant relationship between tachypnea with mortality. These factors should be considered when risk-stratifying patients.

A higher rate of comorbidities (58-86\%) has been reported in this patient group [12,15,19-20]. Common comorbidities include cardiovascular disease, obesity, and diabetes. Early studies from China, Italy, and New York on COVID-19 patients have shown an association of case fatality with medical comorbidities, and 
investigators from Italy demonstrated the potential relationship of comorbidities such as hypertension with mechanical ventilation parameters $[12,15,19-20]$. In the current study, comorbidities were not associated with mortality, however, the cumulative effect of comorbidities in terms of CCI and age were associated with increased mortality [17,20-21]. The lack of direct correlation between types of comorbidities and outcomes could present a type-1 error, again, due to the small sample size of this study.

Once admitted to the ICU, patients suffered a multitude of complications, including ARDS, shock, and renal failure, which increased the risk for mortality similar to other studies [18,21]. The mortality in the present study was $33 \%$. The rates for critically ill COVID-19 patients from other centers are variable, as follows in descending order: 67\% (Seattle, Arentz et al.), 62\% (China, Yang et al.), 50\% (Seattle, Bhatraju et al.), 40\% (New York, Cummings et al.), 39\% (Italy, Grasselli et al.), 39\% (China, Wang et al.), 38\% (China, Huang et al.), 31\% (Atlanta, Sara et al.), and 14\% (California, Ferguson et al.) [11-12,14-18,21-22]. The association of mechanical ventilation, the need for vasopressors, and renal replacement therapy with mortality may simply indicate the effect of the multiorgan failure but also suggests that despite aggressive management, there is a high risk for treatment failure leading to death. Other studies from the US and Italy also indicate a high incidence of ARDS and a high rate of mechanical ventilation (60\%-88\%) [1,11-12,14-15,21,22]. Mechanical ventilation rates were lower in early studies from China: 15\% (Huang et al.), 42\% (Yang et al.), and 47\% (Wang et al.) $[13,16,18]$. This variability could represent the differences in population characteristics, as well as the provision of care, including the use of non-invasive ventilation strategies. There is also a possibility that analyses in several of the above-cited studies included patients who were still admitted to the hospital at the time of the study, resulting in underestimation of mortality and morbidity. Nearly half of patients required oxygen at discharge, however, the long-term need for oxygen is unknown, as is the ultimate impact on pulmonary physiology. Specific aspects of treatment such as strategies used for mechanical ventilation, types, and doses of vasopressors are beyond the scope of this study.

Lastly, since this study represents patients admitted during the earlier phases of COVID-19, treatment strategies did not include remdesivir, interleukin 6 (IL-6) antagonists, convalescent plasma, or dexamethasone, which are now commonly utilized [23]. The higher rate of mortality among those requiring steroids was likely a surrogate for the severity of illness for these patients and is similar to observational studies in pneumonia caused by COVID-19, as well as other phylogenetically similar viruses $[14,19]$. Hydroxychloroquine was used for the majority of patients, and at least in unadjusted comparison, did not appear to impact survival. Another aspect to be explored is the use of non-invasive ventilation (NIV). In an Italian study of critically ill COVID-19 patients, $11 \%$ with acute hypoxemic respiratory failure were managed with NIV [11]. The use and impact of NIV on outcomes were not included in this study.

The limitations of the present study include those inherent to retrospective study design such as recall biases, indication biases, and the presence of uncharacterized confounding factors. While a multivariate regression analysis could have helped identify how various factors impacted mortality in a combined model, it was not performed due to the complexity of the resultant model, which failed to significantly add to the presented results. We plan to perform this in the future with a larger dataset. The follow-up was limited to the inpatient hospital stay and did not capture complications or readmission post-discharge. The data included are from eight different hospitals with a mix of urban academic and rural community hospitals. Variations may exist due to the practice and population patterns of the individual hospitals.

\section{Conclusions}

The present study contributes to the growing body of data on the characteristics and outcomes for COVID19 patients admitted to the ICU. COVID-19 was associated with high mortality and complication rates. Several factors associated with adverse outcomes were identified in the present study. Further investigations are needed to assess the predictive factors that drive mortality to better refine treatment strategies.

\section{Additional Information \\ Disclosures}

Human subjects: Consent was obtained or waived by all participants in this study. Allegheny Health Network (AHN) Research Institute issued approval issued certificate of exemption. Animal subjects: All authors have confirmed that this study did not involve animal subjects or tissue. Conflicts of interest: In compliance with the ICMJE uniform disclosure form, all authors declare the following: Payment/services info: All authors have declared that no financial support was received from any organization for the submitted work. Financial relationships: All authors have declared that they have no financial relationships at present or within the previous three years with any organizations that might have an interest in the submitted work. Other relationships: All authors have declared that there are no other relationships or activities that could appear to have influenced the submitted work.

\section{References}

1. Richardson S, Hirsch JS, Narasimhan M, et al.: Presenting characteristics, comorbidities, and outcomes among 5700 patients hospitalized with COVID-19 in the New York City Area. JAMA. 2020, 323:2052-9. 10.1001/jama.2020.6775 
2. Desai HD, Sharma K, Jadeja DM, Desai HM, Moliya P: COVID-19 pandemic induced stress cardiomyopathy: a literature review. Int J Cardiol Heart Vasc. 2020, 31:100628. 10.1016/j.ijcha.2020.100628

3. CDC. Coronavirus disease 2019 (COVID-19) in the U.S . (2020). Accessed: June 16, 2020: https://www.cdc.gov/coronavirus/2019-ncov/cases-updates/cases-in-us.html.

4. CDC. Coronavirus disease 2019 (COVID-19) . (2020). Accessed: May 6, 2020: https://www.cdc.gov/coronavirus/2019-ncov/need-extra-precautions/racial-ethnic-minorities.html.

5. Wikipedia. Allegheny Health Network. (2020). Accessed: July 5, 2020: https://en.wikipedia.org/wiki/Allegheny_Health_Network.

6. Coronavirus response. Allegheny Health Network . (2020). Accessed: July 5, 2020: https://www.ahn.org/coronavirus.html.

7. Allegheny Health Network starts drive-through testing for COVID-19. (2020). Accessed: July 5, 2020: https://www.wesa.fm/science-health-tech/2020-03-18/allegheny-health-network-starts-drive-throughtesting-for-covid-19.

8. Pennsylvania COVID-19 numbers. Department of Health. (2020). Accessed: May 1, 2020: https://www.health.pa.gov/topics/disease/coronavirus/Pages/Cases.aspx.

9. Ranieri VM, Rubenfeld GD, Thompson BT, et al.: Acute respiratory distress syndrome: the Berlin Definition . JAMA. 2012, 307:2526-33. 10.1001/jama.2012.5669

10. Mehta RL, Kellum JA, Shah SV, Molitoris BA, Ronco C, Warnock DG, Levin A: Acute Kidney Injury Network: report of an initiative to improve outcomes in acute kidney injury. Crit Care. 2007, 11:R31. 10.1186/cc5713

11. Arentz M, Yim E, Klaff L, Lokhandwala S, Riedo FX, Chong M, Lee M: Characteristics and outcomes of 21 critically ill patients with COVID-19 in Washington State. JAMA. 2020, 323:1612-4. 10.1001/jama.2020.4326

12. Grasselli G, Zangrillo A, Zanella A, et al.: Baseline characteristics and outcomes of 1591 patients infected with SARS-CoV-2 admitted to ICUs of the Lombardy Region, Italy. JAMA. 2020, 323:1574-81. 10.1001/jama.2020.5394

13. Wang D, Hu B, Hu C, et al.: Clinical characteristics of 138 hospitalized patients with 2019 novel coronavirus-infected pneumonia in Wuhan, China. JAMA. 2020, 323:1061-9. 10.1001/jama.2020.1585

14. Bhatraju PK, Ghassemieh BJ, Nichols M, et al.: Covid-19 in critically ill patients in the Seattle region - case series. N Engl J Med. 2020, 382:2012-22. 10.1056/NEJMoa2004500

15. Cummings MJ, Baldwin MR, Abrams D, et al.: Epidemiology, clinical course, and outcomes of critically ill adults with COVID-19 in New York City: a prospective cohort study. Lancet. 2020, 395:1763-70. 10.1016/S0140-6736(20)31189-2

16. Huang C, Wang Y, Li X, et al.: Clinical features of patients infected with 2019 novel coronavirus in Wuhan, China. Lancet. 2020, 395:497-506. 10.1016/S0140-6736(20)30183-5

17. Wang Y, Lu X, Li Y, et al.: Clinical course and outcomes of 344 intensive care patients with COVID-19. Am J Respir Crit Care Med. 2020, 201:1430-4. 10.1164/rccm.202003-0736LE

18. Yang X, Yu Y, Xu J, et al.: Clinical course and outcomes of critically ill patients with SARS-CoV-2 pneumonia in Wuhan, China: a single-centered, retrospective, observational study. Lancet Respir Med. 2020, 8:475-81. 10.1016/S2213-2600(20)30079-5

19. Zhou F, Yu T, Du R, et al.: Clinical course and risk factors for mortality of adult inpatients with COVID-19 in Wuhan, China: a retrospective cohort study. Lancet. 2020, 395:1054-62. 10.1016/S0140-6736(20)30566-3

20. Characteristics of and Important Lessons From the Coronavirus Disease 2019 (COVID-19) Outbreak in China: Summary of a Report of 72314 Cases From the Chinese Center for Disease Control and Prevention | Global Health | JAMA | JAMA Network. Accessed July 3. (2020). https://jamanetwork.com/journals/jama/fullarticle/2762130.

21. Auld SC, Caridi-Scheible M, Blum JM, et al.: ICU and ventilator mortality among critically ill adults with coronavirus disease 2019. Crit Care Med. 2020, 48:e799-804. 10.1097/CCM.0000000000004457

22. Ferguson J, Rosser JI, Quintero O, et al.: Characteristics and outcomes of coronavirus disease patients under nonsurge conditions, Northern California, USA, March-April 2020. Emerg Infect Dis. 2020, 26:1679-85. 10.3201/eid2608.201776

23. NIH. What's new. Coronavirus disease COVID-19. COVID-19 treatment guidelines . (2020). Accessed: July 5, 2020: https://www.covid19treatmentguidelines.nih.gov/whats-new/. 